\title{
How Children, Caregivers and Healthcare Professionals Perceive Concussion and Mild Traumatic Brain Injury
}

\author{
Jennifer M. Bain ${ }^{1 *}$, Jeffrey M. Kornitzer², Christin Traba² and Felicia Gliksman ${ }^{4}$ \\ ${ }^{1}$ Department of Child Neurology, New York Presbyterian - Columbia University Medical Center, New York, NY, USA \\ ${ }^{2}$ Department of Child Neurology, Rutgers New Jersey Medical School, Newark, NJ, USA \\ ${ }^{3}$ Department of Pediatrics, Rutgers New Jersey Medical School, Newark, NJ, USA \\ ${ }^{4}$ Department of Child Neurology Hackensack University Medical Center, Hackensack, NJ, USA
}

Received: February 13, 2015; Accepted: March 10, 2015; Published: March 15, 2015

*Corresponding author: Jennifer M. Bain, Department of Child Neurology, 710 West 168th Street, New York, NY 10032, USA, Phone: (212) 305-1338;Email: jb3634@cumc.columbia.edu

\begin{abstract}
The goal of this study was to specify symptoms frequently neglected in concussion. Health care professionals correctly identified $75 \%$ of concussion symptoms, whereas children and caregivers both only correctly identified 31\%. Most people correctly identified headache and ataxia, but many missed photophobia, paresthesias, anxiety and depression. This study demonstrates the general lack of knowledge regarding specific signs and symptoms of concussion.
\end{abstract}

\section{Introduction}

Interest in mild traumatic brain injury, also referred to as concussion, is booming in the public's eye and continues to be an avid area of research for both clinicians and basic scientists. Undoubtedly, these concerns have also been voiced in the pediatric world, as research continues to support the cumulative effect concussion has on the developing brain.

The American Academy of Pediatrics recently published an extensive clinical report in 2010 on management of concussion ${ }^{1}$. In this report, concussion as described as having 5 major features: [1] traumatic etiology, [2] rapid-onset neurologic impairment with spontaneous resolution, [3] functional disturbance, as opposed to structural injury, causing the symptoms, [4] wide spectrum of symptoms, with or without loss of consciousness, that typically resolve sequentially, though postconcussive symptoms may remains, no structural abnormalities on neuroimaging. Resolution of the symptoms typically follow a sequential course; however, it is important to note that in a small percentage of cases, post concussive symptoms may be prolonged." There is also a section on signs and symptoms that are suggestive for traumatic brain injury, divided into 4 sections: physical, cognitive, emotional and sleep. A symptom is defined as a subjective, non-measurable complaint from a patient that is deviation from normal function or feeling, while a sign is an objective indication that may be detected by a physician.

There are several screening tests that have been developed and distributed to coaches and practitioners, including those provided by the American Academy of Neurology, the Colorado Medical Society and Cantu grading systems ${ }^{1}$. A limitation to these tests is they are often time-consuming and may even require training in test administration. Creative attempts to deal with these limitations have included an iPhone application to help coaches and parents identify concussion in a child 2. Interestingly, there remains variability in different modes of evaluation, including differences in self-reporting versus interviewing techniques, as well the gender of interviewers ${ }^{3,4}$. Altogether, there is a lack of a single evaluation that is both highly specific and sensitive for the clinician to screen for concussion symptoms in children.

\section{Methods}

We were interested in determining what symptoms patients, their families, and doctors thought were common after head injury. We surveyed 141 people (42 children, 44 caregivers and 55 health care providers) about their knowledge of concussion symptoms. Subject eligibility for the children included children ages 7 to 21 years of age. The caregivers could be any age and must have had a child who is currently between the ages of 7 and 21 years of age. The healthcare professional was any resident, fellow, attending physician or advanced practice nurse in either the field of neurology or pediatrics. The questionnaire included 25 "yes" or "no" questions whether the symptom was present in concussion. It was administered in the pediatric emergency room, general pediatric clinic or pediatric neurology clinic. Both institutions institutional review boards approved the study.

The common symptoms of concussion were divided into 4 major categories: physical, cognitive, emotional, and sleep. All of the first 22 questions did in fact reflect actual signs and symptoms of concussion; we report the percentage of individuals answering "yes" to each individual questions which reflects that the person correctly identified the concussion symptoms. Questions \#2325 were distractors and not included in the cumulative score for comparison between groups. 


\section{Results}

Overall, health care professionals correctly identified $75 \%$ of the symptoms of concussion, whereas children and caregivers only correctly identified 31\% (Table 1). Even among individual questions, health care professionals consistently identified symptoms at a higher rate than children and caregivers. Between children and caregivers, there was no clear pattern supporting the superiority of one group's responses over the other; children correctly identified concussion symptoms at a higher rate than caregivers for eight of the questions.

There were several patterns, though, that did affect all groups. Physical symptoms of concussion were the most recognized by all groups (Figure 1). Within that category, the majority of all groups correctly identified headache and ataxia. Conversely, a majority of all groups missed that wearing sunglasses inside (photophobia), as well as paresthesias were concussion symptoms. For the symptom of vomiting, there was a wide disparity among groups. The overwhelming majority of health care professionals identified vomiting as a symptom of concussion, while only $17 \%$ of children and $23 \%$ of caregivers did likewise.

Unlike physical symptoms, all groups poorly correlated emotional symptoms of concussion. The vast majority of children and caregivers did not correctly identify anxiety, depression, and other "emotional" symptoms. Interestingly, health care professionals only identified these concussion symptoms $56 \%$ of the time.

\section{Discussion}

With the goal of investigating similarities and differences in knowledge of concussions among children, caregivers, and healthcare professionals, this study may several noteworthy findings. There does seem to be a decent understanding, that physical symptoms such as headache and ataxia could be the result of concussion; however one important exception seems to be vomiting, which was missed by the overwhelming majority of children and caregivers. One unfortunate area of uniform

Table 1: The questions below are taken from the questionnaire provided to study participants. Three questions denoted by asterisk * were distractor questions and not included in data analysis for group totals. Percentages represent the \% of individuals in each group who answered "yes" to the question posed (children $n=29$, caregivers $n=33$, and healthcare professionals $n=49$ ). The total percentages in the final row are based upon all "yes" answers for all questions in the questionnaire except for the distractors denoted by an asterisk.

\begin{tabular}{|c|c|c|c|c|}
\hline & Questionnaire & $\begin{array}{l}\text { Children (\% } \\
\text { answering yes) }\end{array}$ & $\begin{array}{l}\text { Caregivers ( } \% \\
\text { answering yes) }\end{array}$ & $\begin{array}{l}\text { Healthcare Professionals } \\
\text { (\% answering yes) }\end{array}$ \\
\hline \multirow[t]{10}{*}{ Physical } & Having a headache & 62 & 59 & 98 \\
\hline & Feeling dizzy or like the room is spinning around & 59 & 57 & 85 \\
\hline & Feeling "foggy" & 27 & 53 & 93 \\
\hline & Feeling nauseous (like you have to vomit) & 26 & 36 & 91 \\
\hline & Vomiting & 12 & 28 & 91 \\
\hline & Unable to walk straight & 53 & 57 & 80 \\
\hline & $\begin{array}{l}\text { Wearing sunglasses inside or when its cloudy } \\
\text { outside }\end{array}$ & 10 & 12 & 43 \\
\hline & Avoiding loud noises (like the TV or radio) & 44 & 33 & 65 \\
\hline & *Bleeding from the head after injury & 43 & 40 & 38 \\
\hline & *Having a seizure or body shaking uncontrollably & 27 & 42 & 60 \\
\hline \multirow[t]{3}{*}{ Cognitive } & $\begin{array}{l}\text { Taking longer to complete homework } \\
\text { assignments }\end{array}$ & 34 & 36 & 80 \\
\hline & Having a hard time reading a book & 38 & 42 & 82 \\
\hline & Having more trouble reading the blackboard & 30 & 40 & 73 \\
\hline \multirow[t]{4}{*}{ Emotional } & Being angry, irritable or annoyed more easily & 25 & 26 & 73 \\
\hline & Being more sad & 28 & 20 & 49 \\
\hline & Being more easily surprised or "jumpy" & 15 & 7 & 47 \\
\hline & Being more nervous & 22 & 24 & 55 \\
\hline \multirow[t]{3}{*}{ Sleep } & Being more tired during school time & 39 & 23 & 71 \\
\hline & Going to bed early & 29 & 22 & 56 \\
\hline & Sleeping more than usual & 31 & 48 & 84 \\
\hline $\begin{array}{l}\text { Total (excluding } \\
\text { distractors*) }\end{array}$ & & 31 & 34 & 72 \\
\hline
\end{tabular}




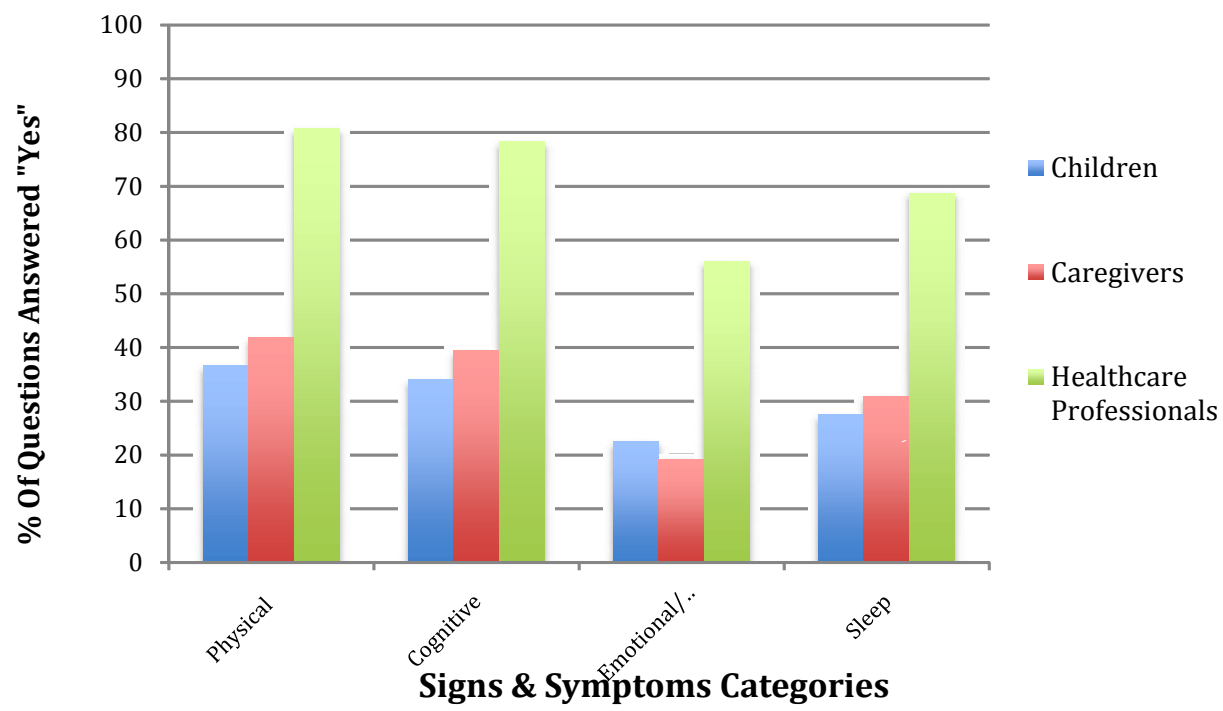

Figure 1: Percentages of correctly identified symptom in the different symptom groups compared between the study groups.

knowledge gap, however, is the emotional symptoms category. Anxiety, depression, and other "emotional" symptoms are underappreciated as symptoms by all groups, including nearly $50 \%$ of healthcare professionals.

These findings represent any important tool in encouraging patient education and the early detection of concussion. While it is axiomatic that healthcare professionals need to understand the symptoms of concussion, child and caregiver knowledge of concussions is likewise imperative. Early detection of concussion can lead to decreased risk of concussion complications, such as second-concussion syndrome

With a growing demand on healthcare professionals to provide care for more patients in less time, it is unrealistic to expect a provider to spend more than several minutes educating a child and family about concussions. A targeted approach to patient and family education could emphasize those areas identified in this study as commonly unknown. For example, given that vomiting is underappreciated as a symptom of concussion, patient and caregiver education might concentrate in emphasizing that vomiting could, indeed, be a symptom of concussion. Similarly, transient feelings of anxiety and depression should also be emphasized as potential symptoms of concussion.. This study demonstrates the lack of knowledge in both our children and their caregivers regarding symptoms of concussion. As clinicians, we should be more aware of the opportunities to teach our patient populations about such symptoms.

\section{References}

1. Halstead ME, Walter KD. Clinical Report-Sport-Related Concussion in Children and Adolescents. Pediatrics. 2010:20805152. doi:10.1542/ peds.2010-2005.

2. Walkinshaw E. iPhone app an aid in diagnosing concussions. CMAJ. 2011;183:21896692. doi:10.1503/cmaj.109-3942.

3. Krol AL, Mrazik M, Naidu D, Brooks BL, Iverson GL. Assessment of symptoms in a concussion management programme: method influences outcome. Brain Inj. 2011;25:22077535. doi:10.3109/0269 9052.2011.624571.

4. Conducting Survey Research. The Health Communication Unit, at the Centre for Health Promotion, University of Toronto. 